\title{
An Archived Differential Evolution Algorithm for Constrained Global Optimization
}

\author{
Zhangjun Huang, Mingxu Ma and Chengen Wang \\ MOE Key Laboratory of Process Industry Automation, Northeastern University, Shenyang, China \\ (Tel : +86-24-83683619; E-mail: zhangjunhuang2000@gmail.com)
}

\begin{abstract}
Many practical engineering problems are naturally global optimization problems with complex constraints and local optimums. However, practicable and effective approaches for those constrained global optimization problems are still insufficient. A modified differential evolution algorithm is put forward for constrained global optimization problems in this work, using a special constraint-handling mechanism based on dynamic penalty functions and fitness calculation of individuals. An archive of solutions is maintained in the evolutionary process so that the best information of previous local optimums can be kept and utilized for the quality estimate of new solutions. Based on the archive of solutions, an iterative control operation is designed in the algorithm to guide the evolutionary process towards a promising space and avoid unnecessary and worthless search processes. Finally, numerical experiments based on a set of eight well-known constrained optimization problems are carried out to investigate the performance of the proposed method, and the experimental results reveal that the proposed algorithm is robust, effective and efficient in solving constrained global optimization problems.
\end{abstract}

Keywords: Constrained optimization, differential evolution, constraint handling, archived solutions, iterative control.

\section{INTRODUCTION}

Due to optimization technology can be great helpful to the improvement of productivity and reduction of resources, it has been successfully applied to a wide range of engineering problems. Many those practical problems have objective functions that are non-differentiable, non-continuous, non-linear, noisy, multi-dimensional or have many local minima and complex constraints because of various practical requirements, but practicable and effective approaches to solve such problems are still far from satisfying and sufficient, and therefore, more valuable work and research on constrained optimization methods are in urgent need for those engineering problems.

In various optimization methods, differential evolution (DE) [1] is a simple but powerful population-based stochastic search technique for solving global optimization problems over continuous spaces, which can be used to find approximate solutions to such engineering problems [2]. Its effectiveness and efficiency have been successfully demonstrated in the last few years through a vast amount of applications [3-4]. However, there are two problems when DE algorithms are utilized for practical problems. One is the way in which the objective function is converted to a suitable fitness function that guides the $\mathrm{DE}$ algorithm towards desired regions of search, and the other is that the final solutions obtained by them are not best optimums but approximate ones or even local minima because of their stochastic nature.

To settle the two problems above, our approach uses a technique based on dynamic penalty functions and fitness calculation of individuals to handle linear and non-linear constraints on the one hand. On the other hand, an archive of solutions is maintained in the evolutionary process so that the best information of previous local optimums can be kept and used for the quality estimate of new solutions. Furthermore, an iterative control operation is designed based on the archive of solutions to guide the search towards a promising space and avoid unnecessary and worthless search processes.

This paper is organized as follows. In Section 2, a review of related works on optimization and $\mathrm{DE}$ techniques is provided. In Section 3, a brief description of the original DE algorithm is given. In Section 4, the description of our approach is presented in detail. The experimental design and obtained results are provided with discussions in Section 5, and some conclusions are established in Section 6.

\section{RELATED WORKS}

Generally, a multi-constrained optimization problem with constraints can be formulated as follows, minimize $f(X)$

subject to

$\begin{array}{ll}g_{j}(X) \leq 0, & j=1, \ldots, m, \\ h_{k}(X)=0, & k=1, \ldots, n, \\ l_{i} \leq x_{i} \leq u_{i}, & i=1, \ldots, D,\end{array}$

where $D$ is the number of design variables, $X=\left(x_{1}, x_{2}, \ldots, x_{D}\right) \in R^{D}$ is the vector of solution, $f$ is the objective function, $m$ and $n$ are the number of inequality and equality constraints respectively. $g_{j}$ and $h_{k}$ are linear or nonlinear real-value functions respectively. $l_{i}$ and $u_{i}$ are the lower and upper bounds of $x_{i}$ respectively, and they define the whole search space $S \subseteq R^{D}$. Inequality and equality constraints define the feasible region as $F=\left\{X \in S \mid g_{j}(X) \leq 0 \wedge h_{k}(X)=0\right\}$

Thus solutions are separated into feasible ones in the feasible region and infeasible ones out the region.

In order to solve such constrained optimization problems accurately and efficiently, many constraint-handling techniques and optimization algorithms have been proposed in previous works [5]. Especially, the DE algorithm has been proposed and generally considered as a reliable, accurate, robust and 
fast optimization method for unconstrained continuous optimization problems [6].

Since the DE algorithm was proposed, it has attracted much attention. Many new versions have been proposed based on the original $\mathrm{DE}$ and applied to many practical optimization cases. Ali and Törn [7] have introduced new versions of $\mathrm{DE}$ algorithm and suggested some modifications to the classical DE. They introduced an auxiliary population of individuals alongside the original population. Sun et al. [8] have introduced a combination of the $\mathrm{DE}$ algorithm and the estimation of distribution algorithm to guide the search towards a promising area by sampling new solutions from a probability model. Liu and Lampinen [9] have introduced a fuzzy adaptive differential evolution algorithm by using fuzzy logic controllers to adapt the search parameters for the mutation operation and crossover operation. Teo [10] has introduced a DE algorithm with a dynamic population sizing strategy called DESAP based on self-adaptation. Becerra and Coello [11] have proposed a cultural algorithm with a differential evolution population by using different knowledge sources to influence the variation operator of the differential evolution algorithm, and obtained their reported results at a relatively low computational cost with their proposed approach on solving constrained optimization problems.

However, there have been very few approaches for handling constraints based on differential evolution [11] Though Storn [3] has proposed constraint adaptation, in which all constraints of the problem at hand are relaxed, so that all individuals in the initial population become feasible, the approach was not suitable for handling equality constraints. Lin et al. [12] have introduced a hybrid differential evolution by using an augmented Lagrangian function with a multiplier updating method to solve constrained problems, where the penalty parameters could be automatically updated so as to obtain a near identical minimum solution despite wide variation in the initial penalty parameters. Montes et al. [13] have proposed a DE-based approach by allowing each parent to generate more than one offspring and using three selection criteria based on feasibility to deal with the constraints, but the approach was not able to solve problems with a dimensionality higher than 22 and more than 11 nonlinear equality constraints. Zielinski and Laur [14] have handled constraints with a modified selection procedure based on a modified selection procedure, but the method failed to reach the best known solutions for four functions of the given 24 test problems.

\section{DIFFERENTIAL EVOLUTION}

$\mathrm{DE}$ is an efficient parallel direct search method that has already shown appealing features for the optimization of continuous space functions [1]. As with all evolutionary optimization algorithms, DE maintains and operates on a population $P$ of constant size $N P$. The population consists of individuals as follows,
$P_{G}=\left(X_{1, G}, X_{2, G}, \cdots, X_{N P, G}\right)$,

where $G$ is the generation number, $N P$ is the population size, and $X_{j, G}$ is the $j$-th individual in population $P_{G}$.

The basic components of this approach are initialization, mutation, crossover and selection operations. In the initialization operation, initial values are selected for control parameters $N P, C R$ and $F$, where $N P$ is the population size, $C R$ is the real-valued crossover rate in $[0,1], F$ is the mutation factor in $[0,2]$. Upper and lower bounds for each design variable are also defined, and a random value is selected within its boundaries for each design variable in an individual of the initial population $P_{0}$ as follows,

$x_{i, j, 0}=l_{i}+\operatorname{rand}_{i}[0,1] \times\left(u_{i}-l_{i}\right)$,

where $i=1, \ldots, D, j=1, \ldots, N P$, and $\operatorname{rand}_{i}[0,1]$ denotes a uniformly distributed random value in $[0,1]$.

The mutation and crossover operations are important operations by which $\mathrm{DE}$ algorithm with different versions is differentiated and identified. The most popular version is the one with the DE-strategy "rand/1/bin", which is used throughout this work, where "rand" indicates that the individuals to be mutated are randomly chosen, "1" is the number of difference vectors used and the "bin" means that a binomial crossover is used [1].

The mutation operation generates a donor vector for each individual of the current population one by one according to the following formulation,

$V_{j, G+1}=X_{r 1, G}+F \times\left(X_{r 2, G}-X_{r 3, G}\right)$,

where $j=1, \ldots, N P, X_{r 1, G}, X_{r 2, G}$ and $X_{r 3, G}$ are three different individuals in the current population, and $\left(X_{r 2, G}-X_{r 3, G}\right)$ denotes the differential item.

In the crossover operation, new trial vector $U_{j, G+1}$ is developed from the elements of the target vector $X_{j, G}$ and the donor vector $V_{j, G+1}$ as follows,

$u_{i, j, G+1}=\left\{\begin{array}{lc}v_{i, j, G+1 !} & \text { if }\left(\operatorname{rand}_{i, j}[0,1] \leq C R\right) \vee\left(i=I_{\text {rand }}\right), \\ x_{i, j, G}, & \text { otherwise, }\end{array}\right.$

where $i=1, \ldots, D, j=1, \ldots, N P, I_{\text {rand }}$ is a random integer from $\{1, \ldots, D\}$.

In the selection operation, the target vector is compared with the trial vector and the one with the lowest function value is admitted to the next generation as follows,

$X_{j, G+1}=\left\{\begin{array}{cc}U_{j, G+1}, & \text { if } f\left(U_{j, G+1}\right) \leq f\left(X_{j, G}\right), \\ X_{j, G^{\prime}} & \text { otherwise, }\end{array}\right.$

where $j=1, \ldots, N P$.

When $\mathrm{DE}$ is running, the mutation, crossover and selection operations will be carried out iteratively until a stopping criterion is reached.

A feature of original $\mathrm{DE}$ algorithm is that three control parameters involved are fixed during the optimization process. However, there still exists a lack of knowledge of how to find reasonably good values for the control parameters of DE for a given function [9].

\section{OUR APPROACH}

In order to solve constrained optimization problems 
effectively and efficiently, we put forward an archived differential evolution (ADE) based on the original DE and some other techniques. The flowchart of the proposed approach is illustrated in Fig. 1.

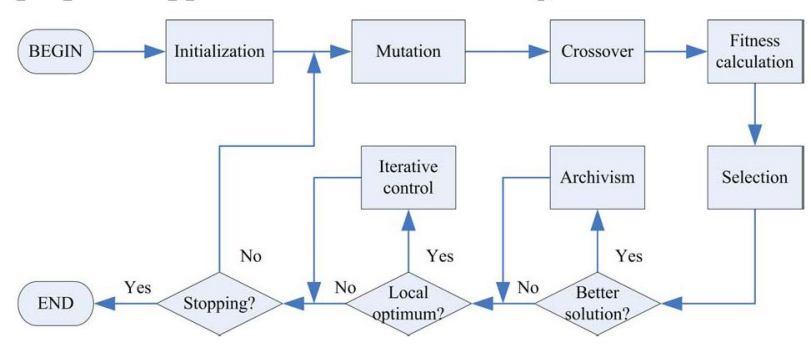

Fig. 1 Flowchart of ADE algorithm.

In brief, we use a special dynamic penalty and fitness function for handling constraints and evaluating individuals in ADE, a modified selection and an archive of solutions for keeping the best solutions at any generation, and an iterative control operation for regulating and guiding the evolutionary process.

\subsection{Constraints handling and individual evaluation}

When DE algorithms are used for solving constrained optimization problems, they require additional mechanisms to handle constraints in their fitness function. The technique of dynamic penalties is one of common constraint-handling techniques, in which the current generation number is involved in the computation of the corresponding penalty item, and so it requires only a few parameters. For separating infeasible individuals from feasible ones, we construct a dynamic penalty function as follows,

$$
p(X)=(C \times G)^{\alpha} \times \sum_{j=1}^{m+n}\left(\max \left\{0, g_{j}(X)\right\}\right)^{\beta},
$$

where $C$ is a adaptable penalty factor, $G$ is the current generation number, $m$ and $n$ are the number of inequality and equality constraints respectively, $\alpha$ and $\beta$ are dynamic penalty exponents, $g_{j}$ denote inequality constraints, including those transformed from equality constraints by

$$
\left|h_{k}(X)\right|-\varepsilon \leq 0 \text {, }
$$

where $k=\{1, \ldots, n\}, \varepsilon$ is the tolerance allowed for equality constraints $h_{k}$.

The dynamic penalty function is used to calculate the fitness of each individual by

$$
\text { fitness }(X)= \begin{cases}-I_{\mathrm{V}}-\frac{p(X)}{p(X)+1}, & \text { if } p(X)>0, \\ -\frac{p(X)}{|p(X)|_{\max }+1}, & \text { otherwise }\end{cases}
$$

where $p$ is the dynamic penalty function, $f$ is the objective function, $|f|_{\max }$ is the maximal objective value for all individuals in the current population, $I_{\mathrm{V}}$ is the number of constraints violated.

Notice that $I_{\mathrm{V}}$ is a positive integer from $\{1, \ldots, m\}$ and an infeasible individual has a positive value of $p$, so its fitness value is a real number in range $\left(-I_{\mathrm{V}}-1,-I_{\mathrm{V}}\right)$. But the fitness value of a feasible individual is in $(-1,1)$, which is higher than that of any infeasible individual.
It is easy to prove that the value of the fitness function goes up with the increase of $p$ in the infeasible region and with the increase of $f$ in the feasible region. Therefore, we can evaluate individuals in the population according to their fitness values, and compare between two individuals by the simple criterion: If an individual has a higher fitness value than another, it is the winner in the comparison, and otherwise it is not.

\subsection{Selection and archivism}

According to the criterion for comparison between individuals, the individual with the highest fitness in the current population can be found and selected as the best individual at current generation.

The archive of solutions is a set of chosen solutions in the previous evolutionary process. The archive is empty at beginning, and the first best solution will be saved to the archive directly. Any new candidate solution at the second or later generation trying entering the archive will be compared with the best one in the archive and only the winner will be archived as follows,

$X_{G, \mathrm{~A}}=\left\{\begin{array}{lc}X_{\mathrm{Best}, G}, & \text { if fitness }\left(X_{\mathrm{Bes}, G}\right)>\text { fitnes }\left(X_{\mathrm{Bes}, \mathrm{A}}\right), \\ X_{\mathrm{Best}, \mathrm{A}}, & \text { otherwise }\end{array}\right.$

where $X_{\mathrm{Best}, G}$ is the best individual at generation $G$, $X_{\text {Best, } \mathrm{A}}$ is the best solution in the archive, fitness is the fitness function, and $X_{G, \mathrm{~A}}$ is the solution to be archived at generation $G$.

If the archived one at generation $G$ is the $X_{\mathrm{Best}, G}$, the archive is considered renewed, otherwise it is not. Thus, the archive keeps all best solutions in previous evolution for the following iterative control.

\subsection{Iterative control}

For estimating whether a solution is near a local optimum, we define and calculate the Euclidian distance between the best individual $X_{\mathrm{Best}, G}$ and the mean individual $X_{\mathrm{Mean}, G}$ at generation $G$ as

$d=\sqrt{\sum_{i=1}^{D}\left(x_{i, \text { Bes }, G}-x_{i, \text { Mean }, G}\right)^{2}}$,

where $x_{i, \text { Best }, G}$ denotes the $i$-th design variable in the best individual $X_{\text {Best }, G}$ at generation $G$, and $x_{i, \text { Mean, } G}$ denotes the $i$-th design variable in the mean individual $X_{\mathrm{Mean}, G}$ that can be calculated by

$x_{i, \text { Mean } G}=\sum_{j=1}^{N P} x_{i, j, G} / N P$,

where $i=1, \ldots, D, j=1, \ldots, N P$.

The best solution of current generation is treated as one near a local optimum if

$d \leq \varepsilon_{d}$,

where $\varepsilon_{d}$ is a small tolerance allowed for the distance.

The iterative control operation will be performed one time if (1) solutions are treated as ones near local optimums continuously and the corresponding counter reaches its maximum, or (2) the archive of solutions is not renewed continuously and the corresponding counter reaches its maximum.

When the iterative control operation is performed, individuals in the next population are generated by 
$x_{i, j, G}=\lambda \times x_{i, \mathrm{Best}, \mathrm{A}}+(1-\lambda) \times\left(x_{i, \mathrm{Best}, \mathrm{A}}-x_{i, j, G-1}\right)$,

where $i=1, \ldots, D, j=1, \ldots, N P, x_{i, \text { Best, } \mathrm{A}}$ is the $i$-th design variable of the best solution in the archive, $x_{i, j, G-1}$ and $x_{i, j, G}$ denote the $i$-th design variable of the $j$-th individual at generation $(G-1)$ and $G$ respectively, $\lambda$ is a random real-valued number in $[0,1]$.

\subsection{Parameters of $\mathrm{ADE}$}

ADE has several parameters to be set beforehand. We list them with some suggestions as follows:

(1) NP. It is the population size and takes a default value of $10 \times D$ in $\mathrm{ADE}$. Larger sizes of population may be adopted, but such values are recommended only for very hard problems if one can afford the extra computational cost.

(2) $G_{\max }$. It is the maximum number of iterations that the algorithm may run. For easy problems, one may set this parameter to 100 or 200 generations. We suggest setting this parameter to 500 for most problems.

(3) $F$ and $C R$. They are the parameters of differential evolution and can be set following the suggestions in [1]. Good default values in $\mathrm{ADE}$ are $F=0.8$ and $C R=0.9$.

(4) $C, \alpha$ and $\beta$. They are three constant parameters in the dynamic penalty function in $\mathrm{ADE}$. The default values for them are commonly $C=0.5, \alpha=2$ and $\beta=2$.

(5) $\varepsilon$ and $\varepsilon_{d}$. $\varepsilon$ is a small tolerance for equality constraint violation allowed when user transforms equalities to inequalities, and $\varepsilon_{d}$ is the tolerance allowed for the distance in Eq. (14) in $\mathrm{ADE}$. The default values for them in $\mathrm{ADE}$ are both set to 0.001 .

\section{NUMERICAL SIMULATION}

\subsection{Experimental results}

Numerical experiments are carried out based on five well-known benchmark functions and three engineering problems to investigate the performances of the proposed ADE. All of the eight test problems, denoted as P1-P8, are taken from [15] and the main characteristics of them are summarized in Table 1, where $D$ is the number of decision variables, LI is the number of linear constraints, NI is the number of nonlinear constraints.

Table 1 Summary of 8 test problems.

\begin{tabular}{cccccc}
\hline Problem & $D$ & $\begin{array}{c}\text { Objective } \\
\text { function }\end{array}$ & LI & NI & $\begin{array}{c}\text { Problem no. } \\
\text { in [15] }\end{array}$ \\
\hline P1 & 13 & quadratic & 9 & 0 & $\mathrm{~g} 01$ \\
P2 & 20 & nonlinear & 1 & 1 & $\mathrm{~g} 02$ \\
P3 & 5 & quadratic & 0 & 6 & $\mathrm{~g} 03$ \\
P4 & 2 & nonlinear & 0 & 2 & $\mathrm{~g} 04$ \\
P5 & 7 & nonlinear & 0 & 4 & $\mathrm{~g} 05$ \\
P6 & 4 & nonlinear & 2 & 5 & $\mathrm{~g} 07$ \\
P7 & 3 & nonlinear & 1 & 3 & g08 \\
P8 & 4 & nonlinear & 3 & 1 & g09 \\
\hline In
\end{tabular}
parameters in $\mathrm{ADE}$ are set as follows: $F=0.8, C R=0.9$, $C=0.5, \quad \alpha=2, \quad \beta=2, \quad \varepsilon=\varepsilon_{d}=0.0001, \quad N P=10 \times D . \quad$ The maximum number of generations is set to 500 for all problems except that for $\mathrm{P} 2$ this parameter is set to 2000 . $\mathrm{ADE}$ is carried out 30 independent runs using the strategy "rand/1/bin" for each test problem.

The approaches applied to P1-P5 include the cultured differential evolution (CDE) [15], the homomorphous mapping (HM) [16], stochastic ranking (SR) [17], the adaptive segregational constraint-handling evolutionary algorithm (ASCHEA) [18], a constraint-handling method for genetic algorithms (TS) [19] and our archived differential evolution (ADE). The results obtained by each approach above for P1-P5 are summarized in Table 2, where the standard deviation (Std. Dev.) is also presented besides the best, the mean and the worst objective values.

As shown in Table 2, the best solutions found by $\mathrm{ADE}$ for P1-P5 are all better than or similar with those obtained by other approaches. The mean and the worst solutions found by $\mathrm{ADE}$ are all better than those obtained by other approaches except for P1 and P2. Though the mean and the worst solutions found by ADE for $\mathrm{P} 1$ and $\mathrm{P} 2$ are not the best in those solutions, they are also not the worst. Furthermore, the standard deviation of the results found by $\mathrm{ADE}$ is very small.

The experimental results for P6-P8 are listed in Table 3 , applying the five approaches: the cultured differential evolution (CDE) [15], GA with co-evolution model (GACO) [20], GA with dominance-based tournament selection (GADTS) [21], co-evolutionary particle swarm optimization (CPSO) [22] and our ADE.

From Table 3 it can be seen that the solutions obtained by ADE for P6-P8 are also better than those found by other compared methods. The best, the mean and the worst solutions found by $\mathrm{ADE}$ are almost same for each of the three problems and even the worst solutions found by $\mathrm{ADE}$ for them are better than the best solutions found by other techniques in the comparison. In addition, the standard deviation of the solutions obtained by $\mathrm{ADE}$ is also the smallest among all considered approaches for the three problems.

\subsection{Discussions}

For all test cases studied here, the search parameters of $\mathrm{ADE}$ were selected rather roughly, and no particular attempts were performed towards optimizing the problem specific adaptation - only the population size and the number of generations was varied from a problem to another. The results in Table 2 and Table 3 have indicated that $\mathrm{ADE}$ succeeded to find feasible and (approximate) optimal solutions for all test cases in all runs, and the best solutions and objective values were better than those obtained by other approaches.

The maximum number of fitness function evaluations (FES) in $\mathrm{ADE}$ is $N P \times G_{\max }$, which varies with the settings of the population size and the maximum number of generations. Thus the maximum number of FES is $130 \times 500=65000$ for P1 and $70 \times 500=35000$ for $\mathrm{P} 5$, while the number of FES is 80000 for P1 and 375000 for P5 in [12].

The evolving process of the objective function values over generations is illustrated in Fig. 2, from which it 
can be seen that $\mathrm{ADE}$ can converge to the global

optimums quickly and steadily for all test problems.

Table 2 Experimental results and their comparisons for P1-P5.

\begin{tabular}{|c|c|c|c|c|c|c|c|}
\hline & roblem & $\mathrm{ADE}$ & CDE [15] & $\mathrm{HM}$ [16] & SR [17] & ASCHEA [18] & TS [19] \\
\hline \multirow{4}{*}{ P1 } & Best & -15.000000 & -15.0000 & -14.7886 & -15.0000 & -15.0 & -15.000 \\
\hline & Mean & -14.983333 & -15.0000 & -14.7082 & -15.000 & -15.0 & -15.000 \\
\hline & Worst & -14.750000 & -15.0000 & -14.6154 & -15.000 & -14.8 & -13.000 \\
\hline & Std. Dev. & 0.063427 & 0.00000 & NA & 0.0 & $\mathrm{NA}$ & NA \\
\hline \multirow{4}{*}{ P2 } & Best & -0.803619 & -0.794669 & -0.79953 & -0.803515 & -0.785 & NA \\
\hline & Mean & -0.793160 & -0.785480 & -0.79671 & -0.781975 & -0.56 & NA \\
\hline & Worst & -0.768791 & -0.779837 & -0.79119 & -0.726288 & -0.59 & NA \\
\hline & Std. Dev. & 0.009672 & 0.003413 & NA & 0.020 & NA & NA \\
\hline \multirow{4}{*}{ P3 } & Best & -30665.538672 & -30665.539 & -30664.5 & -30665.539 & -30665.5 & -30665.537 \\
\hline & Mean & -30665.538672 & -30665.536 & -30655.3 & -30665.539 & -30665.5 & -30665.535 \\
\hline & Worst & -30665.538672 & -30665.509 & -30645.9 & -30665.539 & -30665.5 & -29846.654 \\
\hline & Std. Dev. & $4.966320 \mathrm{E}-8$ & 0.005067 & NA & 0.00002 & NA & NA \\
\hline \multirow{4}{*}{ P4 } & Best & -6961.813876 & -6961.814 & -6952.1 & -6961.814 & -6961.81 & NA \\
\hline & Mean & -6961.813876 & -6960.603 & -6342.6 & -6875.940 & -6961.81 & NA \\
\hline & Worst & -6961.813876 & -6901.285 & -5473.9 & -6350.262 & -6961.81 & NA \\
\hline & Std. Dev. & $1.463572 \mathrm{E}-10$ & 8.474 & NA & 16.0 & NA & NA \\
\hline \multirow{4}{*}{ P5 } & Best & 680.630058 & 680.771 & 680.91 & 680.63 & 680.630 & 680.634460 \\
\hline & Mean & 680.630060 & 681.503 & 681.16 & 680.656 & 680.635 & 680.641724 \\
\hline & Worst & 680.630067 & 685.144 & 683.18 & 680.763 & 680.641 & 680.650879 \\
\hline & Std. Dev. & $2.149568 \mathrm{E}-6$ & 0.67098 & NA & 0.034 & NA & NA \\
\hline
\end{tabular}

$\overline{\mathrm{NA}}=$ not available. A result in boldface means that our approach obtained the same or a better value than any other of the techniques.

Table 3 Experimental results and their comparisons for P6-P8.

\begin{tabular}{|c|c|c|c|c|c|c|}
\hline & oblem & $\mathrm{ADE}$ & CDE [15] & GACO [20] & GADTS [21] & CPSO [22] \\
\hline \multirow{4}{*}{ P6 } & Best & 1.724852 & 1.733461 & 1.748309 & 1.728226 & 1.728024 \\
\hline & Mean & 1.724852 & 1.768158 & 1.771973 & 1.792654 & 1.748831 \\
\hline & Worst & 1.724856 & 1.824105 & 1.785835 & 1.993408 & 1.782143 \\
\hline & Std. Dev. & $6.573240 \mathrm{E}-7$ & 0.022194 & 0.011220 & 0.074713 & 0.012926 \\
\hline \multirow{4}{*}{ P7 } & Best & 0.012665 & 0.0126702 & 0.0127048 & 0.0126810 & 0.0126747 \\
\hline & Mean & 0.012665 & 0.012703 & 0.012769 & 0.0127420 & 0.012730 \\
\hline & Worst & 0.012666 & 0.012790 & 0.012822 & 0.012973 & 0.012924 \\
\hline & Std. Dev. & $6.964692 \mathrm{E}-8$ & 0.000027 & 0.000039 & 0.000059 & 0.000052 \\
\hline \multirow{4}{*}{ P8 } & Best & 6059.714335 & 6059.7340 & 6288.7445 & 6059.946341 & 6061.0777 \\
\hline & Mean & 6060.741397 & 6085.2303 & 6293.8432 & 6177.253268 & 6147.1332 \\
\hline & Worst & 6090.526202 & 6371.0455 & 6308.1497 & 6469.322010 & 6363.8041 \\
\hline & Std. Dev. & 5.625451 & 43.0130 & 7.4133 & 130.929702 & 86.4545 \\
\hline
\end{tabular}

A result in boldface means that our approach obtained the same or a better value than any other of the techniques.

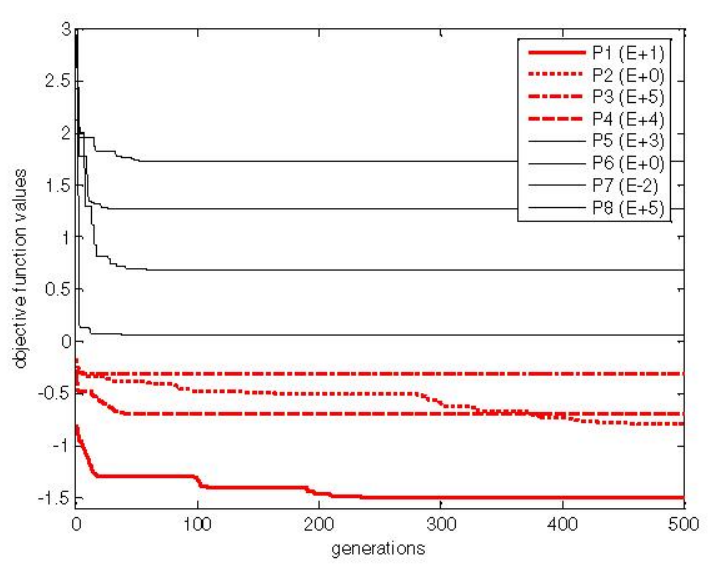

Fig. 2 Objective function values over generations.

\section{CONCLUSION}

A novel archived differential evolution (ADE) for constrained optimization was proposed in this article. The main features of $\mathrm{ADE}$ can be summarized as follows: 1) using a new constraint-handling technique based on the dynamic penalty function and the fitness function of individuals to deal with various constraints effectively; 2) maintaining an archive of solutions to make use of the information of previous solutions and local optimums for the estimation of new solutions; 3) avoiding unnecessary and worthless search processes and speeding up the total convergence by taking advantage of an iterative control operation based on the archive of solutions.

Simulation results based on a set of eight well-known constrained problems and comparisons with previously reported results demonstrated the effectiveness, efficiency and robustness of the proposed ADE. Because of the contribution of archived solutions and the regulation of the iterative control operation, $\mathrm{ADE}$ performed well in terms of FES and outperformed any 
other state-of-the-art approaches referred in terms of the best solutions for all test cases in this paper.

Despite of the encouraging results with the current test problems, only a few conclusions is justified concerning the effectiveness, efficiency and robustness of the proposed method due to the limited size of the test problem set. As part of our future work, we are considering the application of our method to more engineering problems

\section{ACKNOWLEDGEMENTS}

Authors gratefully acknowledge the National Defense Pre-Research Foundation of China (No. 9140A18010106LN0101) and the Doctor Start-up Fund Item of Scientific Office of Liaoning Province of China (No. 20071022) for supporting this research.

\section{REFERENCES}

[1] R. Storn, "Differential evolution - a simple and efficient heuristic for global optimization over continuous spaces," Journal of Global Optimization, Vol. 11, No. 4, pp. 341-359, 1997.

[2] J. Rönkkönen, S. Kukkonen and K. V. Price, "Real-parameter optimization with differential evolution," 2005 IEEE Congress on Evolutionary Computation, Edinburgh, Scotland, September 2005, Vol. 1, pp. 506-513, 2005.

[3] R. Storn, "System design by constraint adaptation and differential evolution," IEEE Transactions on Evolutionary Computation, Vol. 3, No. 1, pp. 22-34, 1999.

[4] A. C. Nearchou and S. L. Omirou, "Differential evolution for sequencing and scheduling optimization," Joumal of Heuristics, Vol. 12, No. 6, pp. 395-411, 2006.

[5] C. A. C. Coello, "Theoretical and numerical constraint-handling techniques used with evolutionary algorithms: a survey of the state of the art," Computer Methods in Applied Mechanics and Engineering, Vol. 191, Nos. 11-12, pp. 1245-1287, 2002.

[6] A. Salman, A. P. Engelbrecht and M. G. H. Omran, "Empirical analysis of self-adaptive differential evolution", European Joumal of Operational Research, Vol. 183, No. 2, pp. 785-804, 2007

[7] M. M. Ali and A. Törn, "Population set-based global optimization algorithms: some modifications and numerical studies," Computers \& Operations Research, Vol. 31, No. 10, pp. 1703-1725, 2004.

[8] J. Sun, Q. Zhang and E. P. K. Tsang, "DE/EDA: a new evolutionary algorithm for global optimization," Information Sciences, Vol. 169, Nos. 3-4, pp. 249-262, 2005.

[9] J. Liu and J. Lampinen, "A fuzzy adaptive differential evolution algorithm," Soft Computing, Vol. 9, No. 6, pp. 448-462, 2005.

[10] J. Teo, "Exploring dynamic self-adaptive populations in differential evolution," Soft Computing, Vol. 10, No. 8, pp. 673-686, 2006.

[11] R. L. Becerra and C. A. C. Coello, "Cultured differential evolution for constrained optimization," Computation Methods in Applied Mechanics and Engineering, Vol. 195, Nos. 33-36, pp. 4303-4322, 2006.

[12] J. Lampinen, "A constraint handling approach for the differential evolution algorithm," 2002 IEEE Congress on Evolutionary Computation, Piscataway, New Jersey, May 2002, Vol. 2, pp. 1468-1473, 2002.

[13] E. M. Montes, J. V. Reyes and C. A. C. Coello, "Modified differential evolution for constrained optimization," 2006 IEEE Congress on Evolutionary Computation, Vancouver, BC, Canada, July 2006, pp. 25-32, 2006.

[14] K. Zielinski and R. Laur, "Constrained single-objective optimization using differential evolution," 2006 IEEE Congress on Evolutionary Computation, Vancouver, BC, Canada, July 2006, pp. 223-230, 2006.

[15] F. Huang, L. Wang and Q. He, "An effective co-evolutionary differential evolution for constrained optimization," Applied Mathematics and Computation, Vol. 186, No. 1, pp. 340-356, 2007.

[16] S. Koziel and Z. Michalewicz, "Evolutionary algorithms, homomorphous mappings, and constrained parameter optimization," IEEE Transactions on Evolutionary Computation, Vol. 7, No. 1, pp. 19-44, 1999.

[17] T. P. Runarsson and X. Yao, "Stochastic ranking for constrained evolutionary optimization," IEEE Transactions on Evolutionary Computation, Vol. 4, No. 3, pp. 284-294, 2000.

[18] S. B. Hamida and M. Schoenauer, "ASCHEA: new results using adaptive segregational constraint handling," 2002 IEEE Congress on Evolutionary Computation, Piscataway, New Jersey, May 2002, Vol. 1, pp. 884-889, 2002.

[19] K. Deb, "An efficient constraint handling method for genetic algorithms," Computer Methods in Applied Mechanics and Engineering, Vol. 186, Nos. 2-4, pp. 311-338, 2000

[20] C. A. C. Coello, "Use of a self-adaptive penalty approach for engineering optimization problems," Computers in Industry, Vol. 41, No. 2, pp. 113-127, 2000.

[21] C. A. C. Coello and E. M. Montes, "Constraint handling in genetic algorithms through the use of dominance-based tournament selection," Advanced Engineering Informatics, Vol. 16, No. 3, pp. 193-203, 2002.

[22] Q. He and L. Wang, "An effective co-evolutionary particle swarm optimization for constrained engineering design problems," Engineering Applications of Artificial Intelligence, Vol. 20, No. 1, pp. 89-99, 2007. 